\title{
Consequence of Heat Transfer on Free Convection Flow of Casson Fluid with Hall Effect
}

\author{
P. Thirunavukarasu, S.Bhuvaneswari, R.Manjula
}

\begin{abstract}
Effect of heat transfer on free convection flow of Casson fluid over a vertical plate with Hall effect has been studied. A similarity analysis method was used to transform the system of partial differential equations describing the problem into an ordinary differential equations, Analytical solutions are obtained by solving the $\mathrm{ODE}$ to analyze the velocity and temperature fields. Variations of interesting parameters on the velocity, heat transfer and skin friction are observed by plotting graphs. Further, it was concluded that the Casson fluid parameter and hall parameter has an retarding influence on velocity profile and also in the skin friction.
\end{abstract}

Index Terms: Casson fluid, Hall effect, MHD, Free convection, Velocity field..

\section{INTRODUCTION}

The MHD of rotating fluids has received considerable attention in the past decades due to its novel features in explaining many natural phenomena in the field of geophysics and astrophysics. The analysis of hydromagnetic flow has its applications in the diverse fields of Science and Technology such as soil sciences, astrophysics, nuclear power reactors etc. Remarkable interest have been achieved in the study of MHD flow problems due to its application in MHD generators, MHD pumps and MHD flow meters etc. Geophysics encounters MHD phenomena in interaction on conducting fluids and magnetic fluids. In the Geophysical problem, the rotating flow of an electrically conducting fluid in presence of magnetic field has got more importance. The study of rotating flow problems is also important in the solar physics dealing with the sunspot development, the solar cycle and the structure of rotating magnetic stars.

Stoke analysed the flow of an incompressible viscous fluid past an impulsively started infinite horizontal plate in its own plane. Chang and Yen [1] have taken the plate to be perfectly conducting in a various transverse magnetic field and analysed. Whenever a strong magnetic field is considered, the effect of Hall current plays a vital role.

Revised Manuscript Received on July 08, 2019.

P. Thirunavukarasu, P. G. and Research Department of Mathematics, Periyar E.V.R. College (Autonomous), Tiruchirappalli-620 023, India.

S.bhuvaneswari, p. G. and Research Department of Mathematics, Kamban College Of Arts And Science for Women, Tiruvannamalai, India.

R.manjula, npa centenary polytechnic college, Sakthi Hills, Kotagiri, The Nilgiris-643 217
Kurzweg [2] showed that the novel enhanced heat transfer process under discussion can also occur in the classical Stoke's problem of oscillating flat plate immersed within a viscous fluid of infinite extent when a constant temperature gradient is superimposed on the fluid parallel to the direction of motion of the plate. He has calculated the thermal flux boundary layer thickness as a function of Prandtl number. Okelo [3] investigated the unsteady free convection incompressible fluid past a semi-infinite vertical porous plate in the presence of a strong magnetic field inclined at an angle to the plate with Hall effect. The effect of Hall and rotation on heat transfer MHD flow of oscillating fluid in a porous channel was analyzed by Khen Chand [4]. Sundarnath [5] studied the effects of Hall current on MHD flow past an accelerated plate with heat transfer. Unsteady boundary layer flow of a Casson fluid due to an impulsively started moving flat plate was investigated by Mustafa et.al. [6]. But so far, no attempt has been made on the effect of heat transfer in Casson fluid flow with hall current where the plate is uniformly accelerated, which forms the main part of this chapter.

Heat transfer concerned with two things: temperature and flow of heat. Temperature represents the thermal energy available while heat flow represents the movement of thermal energy from place to place. So far, no attempt has been made to study explicitly and extensively the effect of uniformly accelerated plate over the enhancement of heat transfer with magnetic field for a Casson fluid. Hence the purpose of the present study is to analyze the effect of heat transfer on free convection flow of Casson fluid over a uniformly accelerated plate with Hall effect and rotation.

\section{Formulation of the Problem}

Consider an incompressible electrically conducting, Casson fluid past an infinite vertical flat plate occupying the plane $y$ $=0$. The ${ }^{x}$-axis is taken in the direction of the motion of the plate and ${ }^{z}$-axis lying on the plate normal to both ${ }^{x}$ and $\mathrm{y}-$ axis. Initially it is assumed that the plate and the fluid rotate in unison with a uniform angular velocity $\bar{\Omega}$ about the y - axis normal to the plate are at the same temperature $T$ everywhere in the fluid. At time

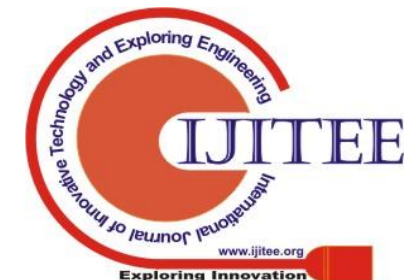


$t>0_{v}$ the plate starts moving impulsively with the uniform velocity in its own plane along the ${ }^{x}$-axis. Also the temperature of the plate is raised/lowered to $T_{\infty}{ }^{\circ}$ A uniform

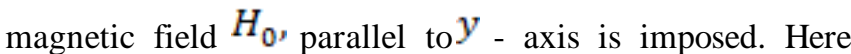

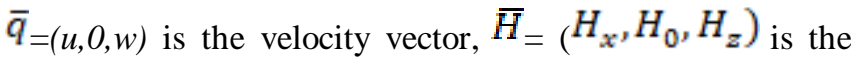
magnetic induction, $\quad \bar{\Omega}_{=}\left(0,{ }^{\Omega}, 0\right)$ denotes uniform angular velocity and $\bar{E}=\left(E_{x,}, 0, E_{z}\right)$ is the electro static field. The rheological equation of state for an isotropic and incompressible flow of a Casson fluid is as follows [7]

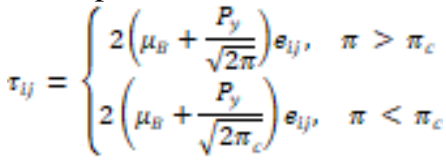

where $\pi=e_{i j} e_{i j}$ and $e_{i j}$ are the $(i, j) t h$ component of the deformation rate, $\pi$ is the product of the component of deformation rate with itself, $\pi_{\varepsilon}$ is a critical value of this product based on the non-Newtonian model, $\mu_{B}$ is plastic dynamic viscosity of the non-Newtonian fluid and $P_{y}$ is the yield stress of the fluid. With the above assumptions, in the absence of an input electric field the governing boundary layer equations with Boussinesq's approximation are considered by neglecting viscous and Joules dissipation.

$$
\begin{aligned}
& \nabla \cdot \bar{q}=0
\end{aligned}
$$

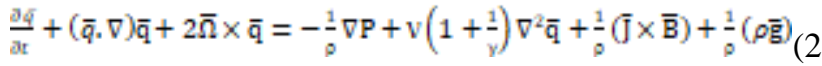

$$
\begin{aligned}
& \rho C_{p}\left[\frac{\partial T}{\partial t}+(\bar{q} \cdot \nabla) T\right]=K \nabla^{2} T \\
& \frac{\overline{\mathrm{I}}}{\sigma}=(\overline{\mathrm{E}}+\bar{q} \times \overline{\mathrm{B}})-\frac{\overline{\mathrm{J} \times \overline{\mathrm{B}}}}{\text { ne }}(4)
\end{aligned}
$$$$
\text { ) }
$$

where ${ }^{P}$ is the pressure, $\rho$ is the density, $v_{\text {is kinematic }}$ viscosity, $\overline{\mathrm{J}} \times \overline{\mathrm{B}}$ is the Lorentz force, $Y$ is the Casson fluid parameter, $\overline{\mathrm{g}}$ is the acceleration due to gravity, ${ }^{C}$ is the specific heat at constant pressure, $K$ is the thermal conductivity, $\overline{\mathrm{I}}$ is the current density and $\sigma$ is the electrical conductivity. Now using, $T(y, t)-T_{\infty}=\theta_{T}(y, t)$, where $T(y, t)$ is the temperature of the fluid in the boundary layer and $T_{\infty}$ is the temperature of the fluid far away from the plate.

Subject to the boundary conditions,

$$
\begin{aligned}
& u=0, w=0, \theta_{T}=0 \text { for all } t \leq 0 \text { and for all } y \\
& u=U_{0 a} w=0, \theta_{T}(0, t)=w e \text { for all } t>0 \text { and } y=0 \\
& u \rightarrow 0, w \rightarrow 0, \theta_{T} \rightarrow 0 \text { for all } t>0 \text { and } y \rightarrow \infty(5)
\end{aligned}
$$

At infinity the magnetic induction is uniform with components $\left(0, H_{0,0}\right)$ and hence the current density vanishes and since the free stream is at rest, it follows from generalized Ohm's law that $\overline{\mathrm{E}}=0$ as $y \rightarrow \infty$. Assuming small magnetic Reynolds number for the flow, the induced magnetic field is neglected in comparison to the applied constant field $H_{0}$ *

Let us introduce the following non-dimensional quantities:

$$
\begin{aligned}
& y^{*}=\frac{U_{0} \cdot y}{v}, u^{*}=\frac{u}{U_{0}}, w^{*}=\frac{w}{U_{0}{ }^{\prime}} \\
& t^{*}=\frac{U_{0}^{2} t}{v}, \theta_{T}^{*}=\frac{\theta_{T}}{a}, G r=\frac{g \beta v a}{U_{0}^{3}}, \\
& m=\omega \tau_{y} M^{2}=\frac{\sigma H_{0}^{2} v}{\rho U_{0}^{2}}, \\
& \operatorname{Pr}=\frac{v \rho c_{p}}{K}, K^{2}=\frac{v_{y}}{U_{0}^{2}}, \xi=\frac{v \omega}{U_{0}^{2}}
\end{aligned}
$$

The non-dimensional forms of equations (1), (2) and (3) (dropping the stars) are

$$
\begin{aligned}
& \frac{\partial u}{\partial t}=\left(1+\frac{1}{Y}\right) \frac{\partial^{2} u}{\partial y^{2}}+G r \theta_{T}-\frac{M^{2}}{\left(1+m^{2}\right)}(u+m w)-2 w K^{2} \\
& \frac{\partial w}{\partial t}=\left(1+\frac{1}{Y}\right) \frac{\partial^{2} W}{\partial y^{2}}+\frac{M^{2}}{\left(1+m^{2}\right)}(m u-w)+2 u K^{2} \\
& \frac{\partial^{2} \theta_{T}}{\partial y^{2}}=\operatorname{Pr} \frac{\partial \theta_{T}}{\partial t}
\end{aligned}
$$

The corresponding boundary conditions (5) in non-dimensional form are

$t \leq 0, u(y, t)=w(y, t)=0, \theta_{T}(y, 0)=0$ for all $y$

$t>0: u(0, t)=1_{v} W(0, t)=0, \theta_{T}(0, t)=e^{i k t}$

$t>0: u(y, t) \rightarrow 0, w(y, t) \rightarrow 0, \theta_{T}(y, t) \rightarrow 0$ as $y \rightarrow \infty$

(10)

\section{Solution of the Problem}

The equations (7) and (8) can be combined using the complex function $q=u+i w$

$$
\begin{aligned}
& \frac{\partial q}{\partial t}=c \frac{\partial^{2} q}{\partial y^{2}}-\left[\left(\frac{M^{2}}{1+m^{2}}\right)(1-i m)-2 i K^{2}\right] q+G r \theta_{T} \\
& \text { where } c=\left(1+\frac{1}{\gamma}\right)
\end{aligned}
$$

The boundary condition (10) are transformed to $q(y, 0)=0, \quad q(0, t)=1_{v} \quad q(\infty, t)=0$

$\theta_{T}(y, 0)=0, \quad \theta_{T}(0, t)=e^{i k t}, \quad \theta_{T}(\infty, t)=0$

Substitute $\theta_{T}(y, t)=e^{i k t} f(y)$ in equation (9), we have

$f^{s y}(y)-i \xi \operatorname{Pr} f(y)=0$

The equation (13) can be solved under the boundary condition

$f(0)=1, f(\infty)=0$

Hence the solution is $f(y)=e^{-y \sqrt{4 p_{P y}}}$

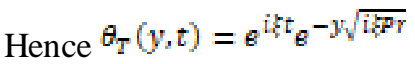

Separating the real and imaginary parts and taking the real part only, we get

$\theta_{T_{p}}(y, t)=e^{-y x_{1}} \operatorname{Cos}\left(\xi t-L_{1} y\right)(16)$

where $L_{1}=\sqrt{\frac{F P_{Y}}{2}}$

Also substitute $q\left(y_{s} t\right)=e^{i k t} g(y)$ in (11), we have $g^{x y}(y)-\frac{(i k+\beta)}{c} g(y)=-\frac{G Y}{c} e^{-3 \sqrt{L k P Y}}$

Here $\beta=a+i b \quad$ where $a=\frac{M^{2}}{1+m^{2}}$ and $b=\frac{-M^{2} m}{1+m^{2}}-2 K^{2}$ 
The equation (17) can be solved under the boundary conditions,

$$
g(0)=e^{-i p t} g(\infty)=0
$$

The solution is obtained and Separating $q(y, t)$ into real and imaginary parts,

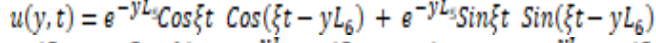

$$
\begin{aligned}
& +\frac{(\xi \operatorname{Prc}-\xi-b) G r e^{-y L_{2} \operatorname{Sin}\left(\xi t-y L_{\overline{6}}\right)}}{(\xi \operatorname{Prc}-\xi-b)^{2}+a^{2}}-\frac{a G r e^{-y L_{2} \operatorname{Cos}\left(\xi t-y L_{6}\right)}}{(\xi \operatorname{Prc}-\xi-b)^{2}+a^{2}}
\end{aligned}
$$

$$
-\frac{(\xi \operatorname{Prc}-\xi-b) G r e^{-y L_{1} \operatorname{Sin}\left(\xi t-y L_{1}\right)}}{(\xi \operatorname{Prc}-\xi-b)^{2}+a^{2}}+\frac{a G r e^{-y L_{1}} \operatorname{Cos}\left(\xi t-y L_{1}\right)}{(\xi \operatorname{Prc}-\xi-b)^{2}+a^{2}}
$$

$w(y, t)=e^{-y L_{1}} \operatorname{Cos} \xi t \operatorname{Sin}\left(\xi t-y L_{6}\right)-e^{-y L_{-} \operatorname{Sin} \xi t \operatorname{Cos}\left(\xi t-y L_{6}\right)}$

$$
\begin{aligned}
& -\frac{(\xi \operatorname{Prc}-\xi-b) G r e^{-y L} \operatorname{Cos}\left(\xi t-y L_{6}\right)}{(\xi \operatorname{Prc}-\xi-b)^{2}+a^{2}}-\frac{a G r e^{-y L_{2} \operatorname{Sin}\left(\xi t-y L_{6}\right)}}{(\xi \operatorname{Prc}-\xi-b)^{2}+a^{2}} \\
& +\frac{(\xi \operatorname{Prc}-\xi-b) G r e^{-y L_{1} \operatorname{Cos}\left(\xi t-y L_{1}\right)}}{(\xi \operatorname{Prc}-\xi-b)^{2}+a^{2}}+\frac{a \operatorname{Gr} e^{-y L_{1} \operatorname{Sin}\left(\xi t-y L_{1}\right)}}{(\xi \operatorname{Prc}-\xi-b)^{2}+a^{2}} \\
& \text { where } L_{2}=\sqrt{a^{2}+(\xi+b)^{2}} ; L_{3}=\sqrt{\frac{a+x_{3}}{2}} L_{4}=\sqrt{\frac{-a+x_{2}}{2}} ; \\
& L_{5}=\frac{L_{4}}{\sqrt{6}} L_{6}=\frac{L_{4}}{\sqrt{c}}
\end{aligned}
$$

\section{Shearing STRESS AND NuSSElt NUMbER}

Knowing the velocity field, the skin friction at the plate can be obtained, which in non-dimensional form is given by

$$
\begin{aligned}
& \tau_{x}=-L_{3}+S_{1}\left[-\sin \xi t L_{a}-L_{4} \cos \xi t\right]-S_{2}\left[-\cos \xi t L_{3}+\right. \\
& \left.L_{4} \sin \xi t\right] \\
& -S_{1}\left[-\sin \xi t L_{1}-L_{1} \cos \xi t\right]+S_{2}\left[-\cos \xi t L_{1}+\sin \xi t L_{1}\right] \\
& \text { and } \\
& \tau_{z}=-L_{4}-S_{1}\left[\sin \xi t L_{4}-L_{3} \cos \xi t\right]-S_{2}\left[-\cos \xi t L_{4}-L_{3} \sin \xi t\right] \\
& +S_{1}\left[\sin \xi t L_{4}-L_{1} \cos \xi t\right]+S_{2}\left[-\cos \xi t L_{1}-\sin \xi t L_{1}\right]
\end{aligned}
$$

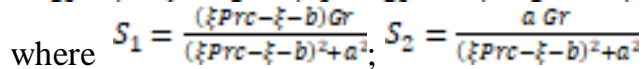

Knowing the temperature field, the rate of heat transfer coefficient can be obtained, which in the non-dimensional form, in terms of the Nusselt number $(N u)$ is given by,

$$
N u=-e^{i \xi t}[\sqrt{i \xi P r}+\xi t]
$$

\section{RESUlTS AND DISCUSSION}

The effect of the Hartmann number $M^{2}$, Hall parameter $m$, Rotation parameter $K^{2}$, Prandtl number ${ }^{P r}$, Casson fluid parameter $\gamma$ and Grashof number $G r$ in the velocity components $u$ and $w$ are illustrated in the figures for the clear understanding of the problem.

Figure 1 show the variation of the primary ${ }^{u}$ and secondary $w$ velocities with increase in the Hartmann number $M^{2}$. Primary velocity ${ }^{u}$ decreases with increase in the Hartmann

number $M^{2}$ in the boundary layer which shows the general tendency of Lorentz force has oppose the motion of an electrically conducting fluid. But the magnitude of the secondary velocity ${ }^{W}$ increase with increase in Hartmann number within the thin boundary layer near the plate boundary and then flow that get reversed behaves as a primary velocity in presence of magnetic field. Figure 2 depict both the velocity profiles when Hall parameter increased. From figures it is clear that, the increase in Hall parameter have a retarding influence on the primary and secondary velocities.

In figures 3 , the primary and secondary velocity profile for various values of thermal Grashof number are presented. Grashof number is used in analyzing the velocity distribution in natural convection. Grashof number is the ratio of buoyancy force due to the spatial variation in the fluid density to the viscous force. Grashof number in positive sense indicates the cooling effect near the plate. From figure it is clear that, the primary velocity and the secondary velocity are highly influenced, so it is accelerated in its flow.

The influences of the Prandtl number on primary and secondary velocities are shown in figures 4 . Prandtl number being the ratio of momentum diffusivity to thermal diffusivity will influence the fluid flow as long as the velocity field and the temperature field are coupled. Hear, the primary velocity increase rapidly near the wall and gradually reduces to the free stream velocity. The secondary velocity gradually decreases with the increase in values of Prandtl number.

The influence of the Casson fluid parameter on the velocity profiles are shown in figures 5. It is observed that both the velocity decreases with increasing values of Casson fluid parameter. It is important to note that an increase in Casson parameter makes the velocity boundary layer thickness shorter. Figure 6 show that the primary velocity component decreases and secondary velocity component increase, when time is increased.

In figure 8 , the temperature profile for different values of time is presented. The size of the thermal boundary layer increases with decreasing Prandtl number because the Boussinesq's approximation in the momentum equation consists of assuming that density of the fluid varies with temperature linearly. Since the velocity and temperature are coupled. Similar type of behavior will be seen both in velocity and temperature. Here temperature profile gradually reduces to reach the free stream temperature. Non-dimensional viscous shear stresses $\tau_{x}, \tau_{z}$ (skin friction) at the boundary $y=0$ due to the primary and secondary flows with control parameters are shown in figures 7 . Shearing stress ${ }{ }_{x}$ and

$\tau_{z}$ decrease as the value of Casson fluid parameter increases

\section{CONCLUSION}

From the above results and discussion following conclusions are made. It is 
elucidated that the effect of Hall parameter and Casson fluid parameter decreases both the fluid velocity components whereas the Hartmann number and Grashof number decreases primary velocity and increases secondary velocity. It is noted that the increase in Prandtl number increases primary velocity but decreases secondary velocity. It is concluded that the temperature profile decreases when time increases. With respect to Hall parameter both the shear stress decreses with increase of Casson fluid parameter.
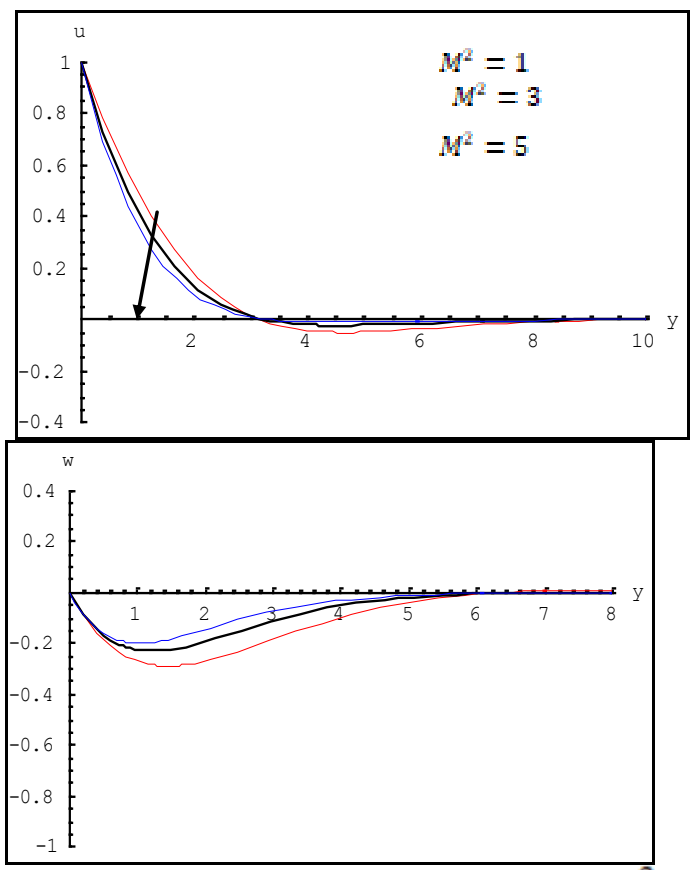

Figure 1 : Effect of Hartmann number $\left(M^{2}\right)$ on primary and secondary velocity
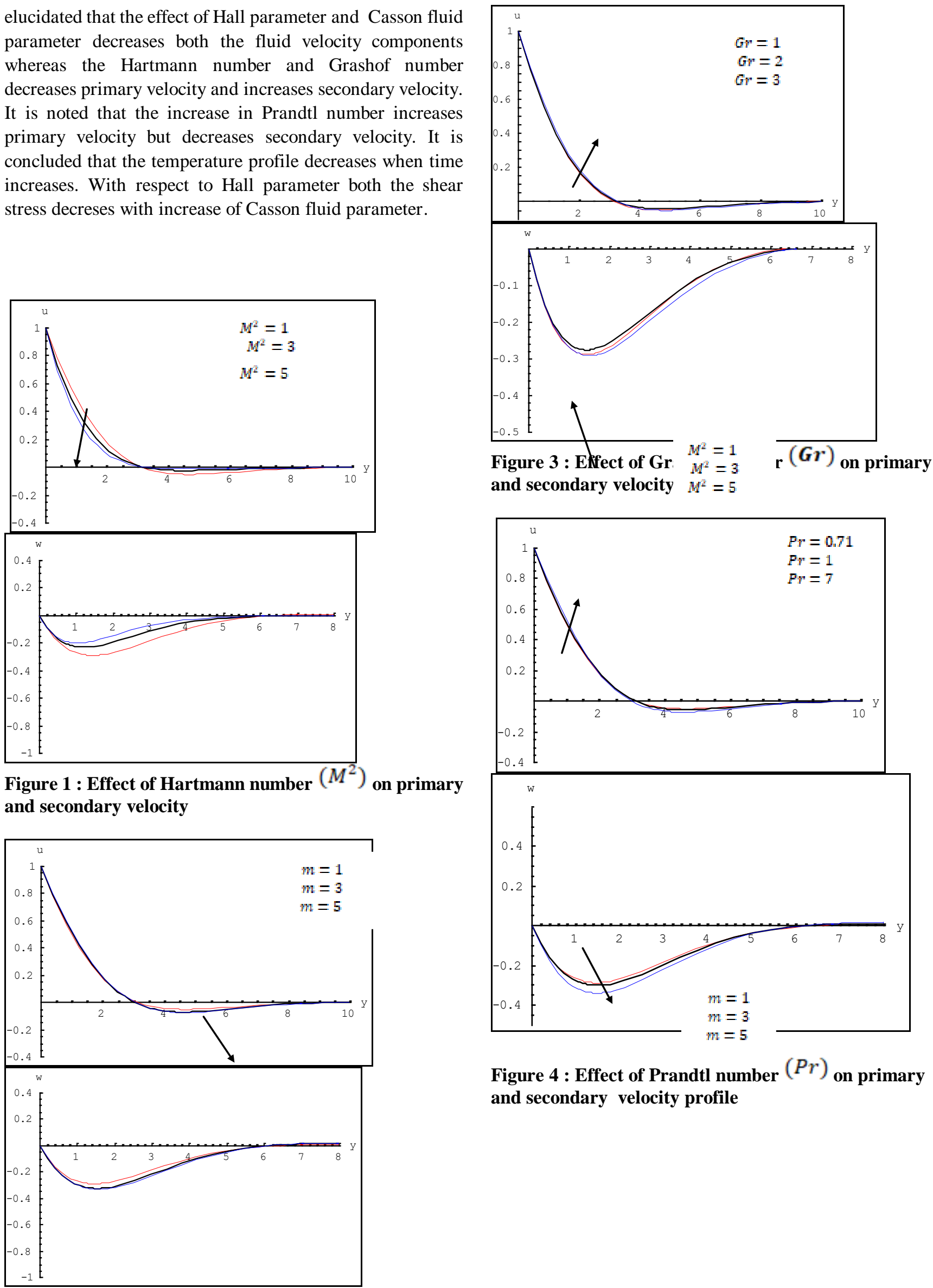

Figure 4 : Effect of Prandtl number $(P r)$ on primary and secondary velocity profile

Figure 2 : Effect of Hall parameter $(m)$ on primary and secondary velocity profile 


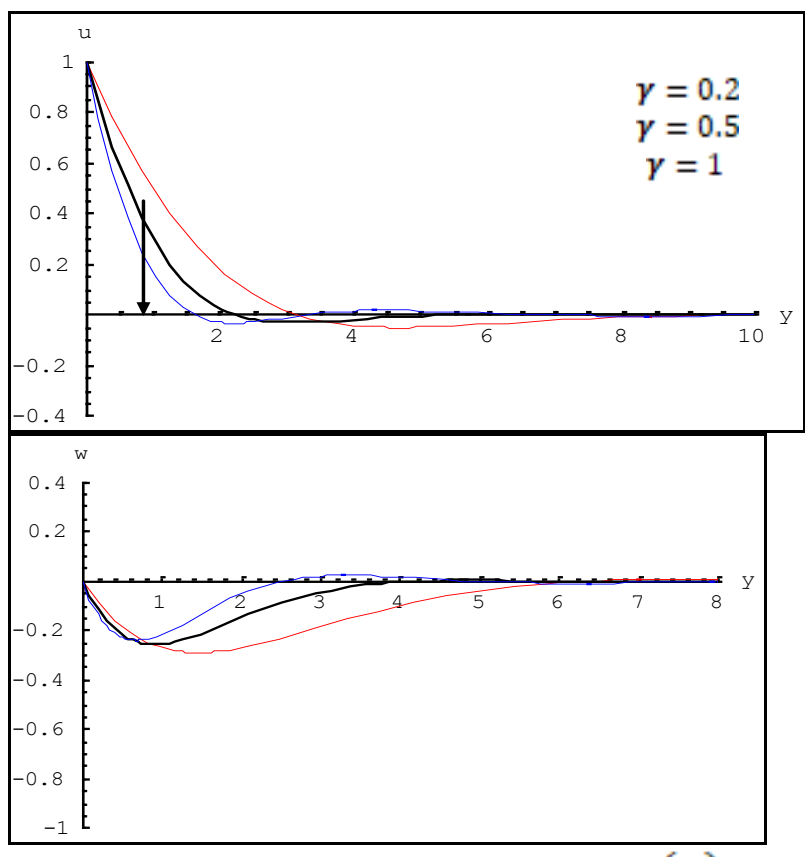

Figure 5 : Effect of Casson fluid parameter $(\gamma)$ on primary and secondary velocity profile when $t=1 ; \operatorname{Pr}=0.71 ; M^{2}=1 ; K^{2}=2 ; G r=2 ; \xi=t ; n 1 ;=K_{1}^{2}=2 ; M^{2}=2 ; G r=2 ; \xi=1 ; \operatorname{Pr}=0.71$

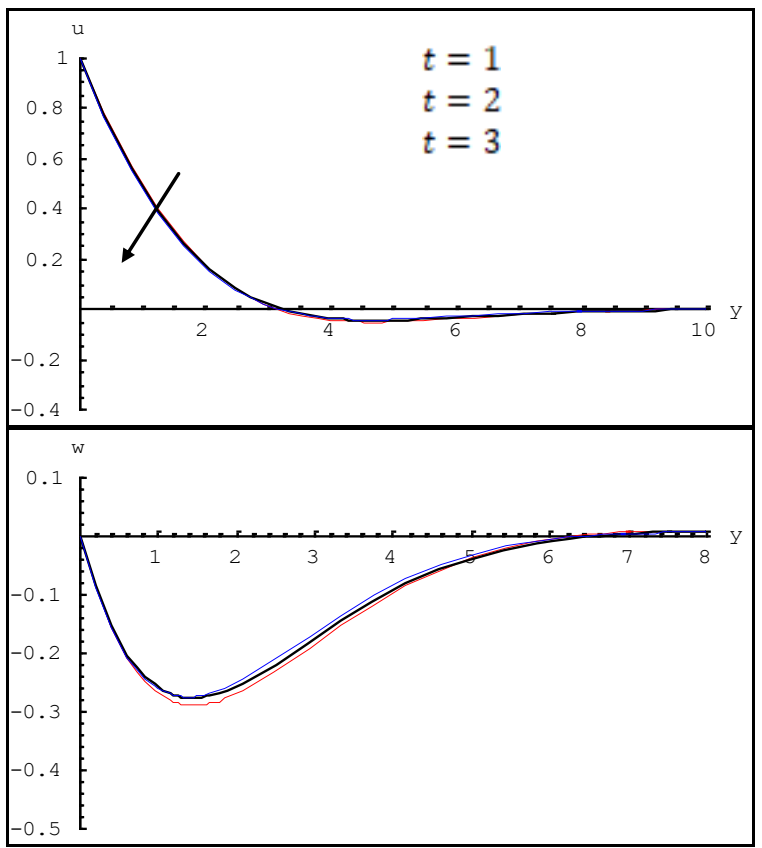

Figure 6 :Effect of time $(t)$ on primary and secondary velocity profile

when

$K^{2}=1 ; \operatorname{Pr}=0.71 ; m=1 ; M^{2}=2 ; G r=2 ; \xi=1 ;$

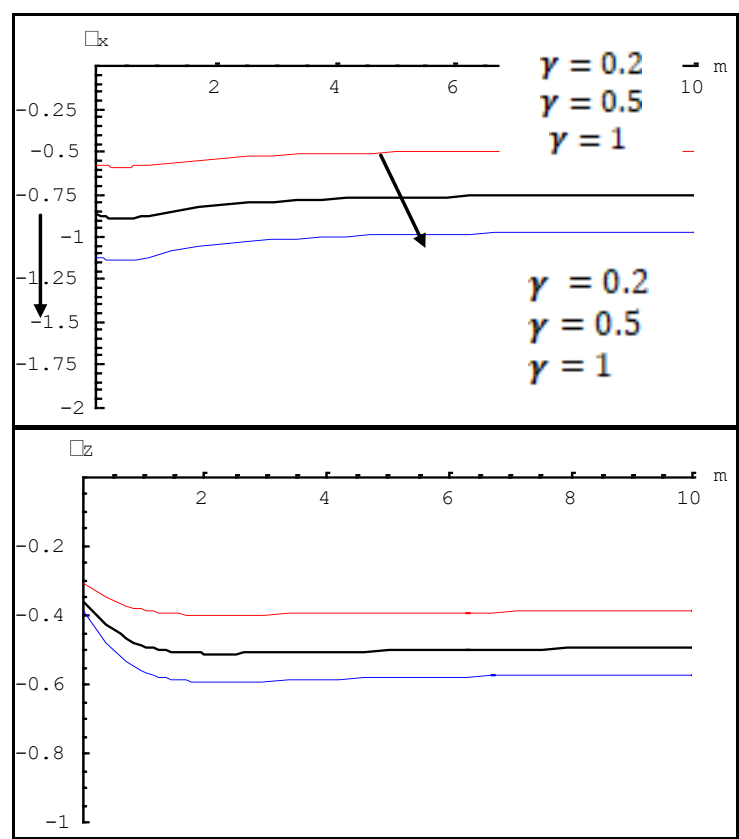

Figure 7 : Shear stress $\boldsymbol{\tau}_{x}$ and $\boldsymbol{\tau}_{z \text { for different }} \gamma$ when

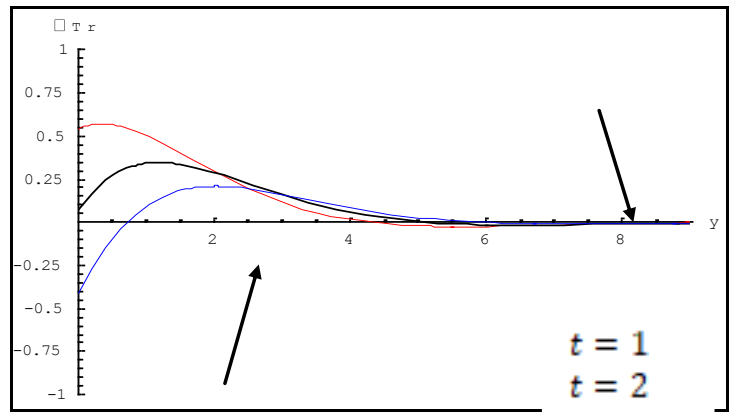

Figure 8 : Temperature profile for $1=3$ es of time (t) when $\xi=1 ; P_{r}=0.71$

\section{REFERENCES}

1. Chang. C and Yen. J, 1959. Rayleigh's Problem in Magnetohydrodynamics, Phys. Fluids, 2: 239

Kurzweg V. Marple, 1988. United States Court of Appeals, Fifth Circuit 7, No. 87- 3615.

2. Okelo, J.A., 2007. Heat and Mass transfer past a semi infinite vertical porous plate in MHD flows, Ph.D., Dissertation, Jomo Kenyatta University of agriculture and technology.

3. Khen Chand, Singh, K.D. and Shavnam Sharma, 2013. Effect of Hall current and rotation on heat transfer in MHD flow of oscillating dusty fluid in a porous channel, Indian Journal of pure and applied physics, 51, 669-682.

. Sundarnath. J.K. and Muthucumarswamy, R., 2015. Hall effect on MHD flow $=2$ accelerated plate with heat transfer, Int. J. of applied mechanics and engineering, 20(1): 171-181.

5. Mustafa, M., Hayat, T., Pop, I. and Aziz, A, 2011. Unsteady boundary layer flow of a Casson fluid due to an impulsively started moving flat plate, Heat Transfer Asian Res. 40(6):563-576.

6. Casson, N, 1959. A flow equation for Pigment -oil suspension of the priniting ink type. In: Mill, C.C., Ed., Rheology of disperse systems, Pergamon press, Oxford, 84-104. 


\section{AUTHORS PROFILE}

P. THIRUNAVUKARASU P. G. and Research Department of Mathematics, Periyar E.V.R. College (Autonomous), Tiruchirappalli-620 023, India.

S.BHUVANESWARI P. G. and Research Department of Mathematics, Kamban College Of Arts And Science for Women, Tiruvannamalai, India.

R.MANJULA NPA centenary polytechnic college, Sakthi Hills, Kotagiri, The Nilgiris-643 217. 